\title{
Advanced glycation in diabetes, aging and age-related diseases: editorial and dedication
}

Vincent M Monnier $^{1} \cdot$ Naoyuki Taniguchi $^{2}$

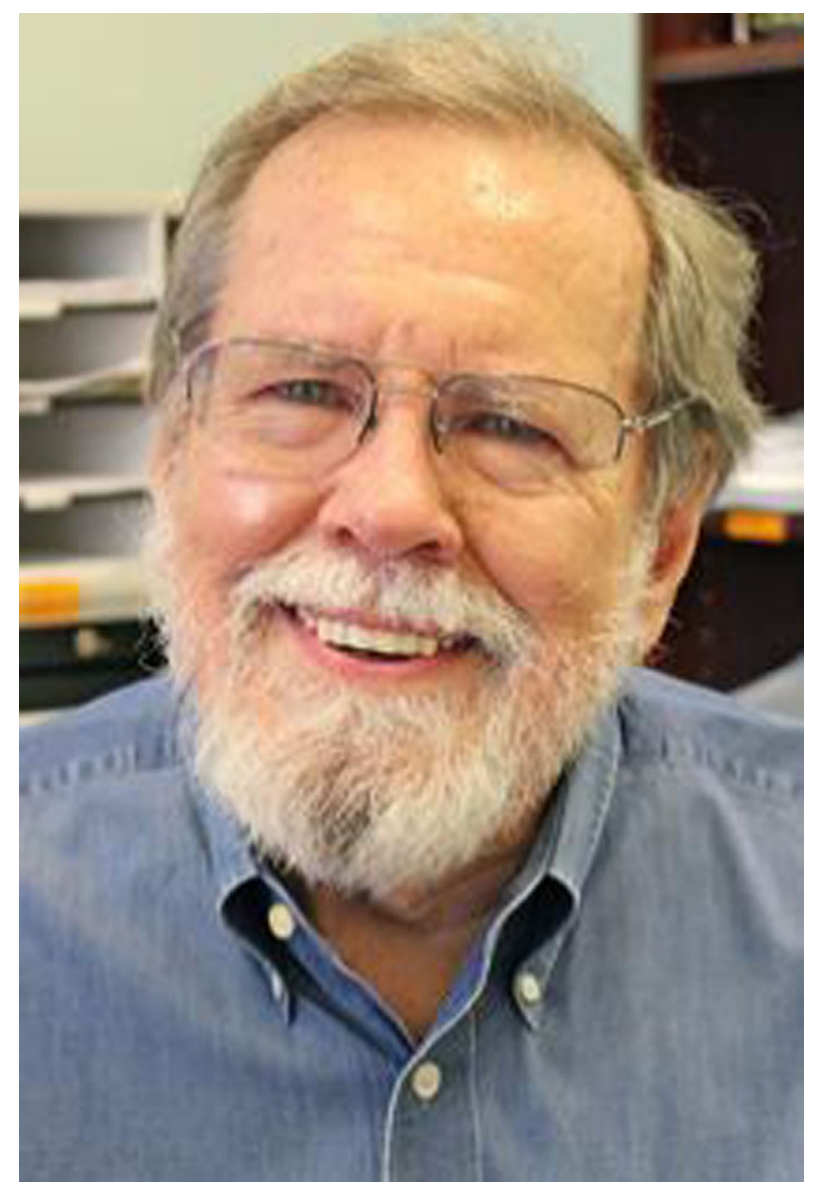

John W. Baynes, PhD.

Carolina Distinguished Professor Emeritus.

"A glycochemist who became a pioneer of the Maillard Reaction in Vivo".

$\triangle$ Vincent M Monnier

vmm3@cwru.edu

Case Western Reserve University, Cleveland, OH, USA

2 RIKEN, Wako, Saitama, Japan 


\section{Editorial and dedication}

This Special Issue on "Advanced Glycation in Diabetes, Aging and Age-Related Diseases" is dedicated to a giant and pioneer in the field of the Maillard Reaction (MR) in vivo, John W. Baynes PhD, in honor of his 75th birthday, his retirement from benchwork research, and in particular in celebration of his enormous accomplishments in Science to date.

Following service in the Army, from which he was discharged with distinction ("Meritorious Service" award and Bronze Star Medal), John Baynes' scientific career started in 1967 with a Master's Thesis in Organic Chemistry with Prof. Arthur R. Lepley at Marshall University where he studied alkylation reactions initiated by interaction of organo-metallics and alkylhalides. He obtained his PhD in 1973 from Johns Hopkins University with Prof. Edward C. Heath for studies on the biosynthesis of glycoproteins with emphasis on the role of lipid intermediates in the enzymatic glycosylation of mammalian glycoproteins. These were among the earliest studies on the dolichol pathway which led to characterization of mannose-P-dolichol and oligosaccharide pyro-phosphoryl glycolipid intermediates involved in glycoprotein biosynthesis. From 1973 to 1976, at the University of Minnesota, John Baynes did postdoctoral studies in Clinical Chemistry and postdoctoral research with Dr. Finn Wold, at the Department of Biochemistry, on the role of carbohydrates in the clearance of glycoproteins from the circulation. These studies with isozymes of RNase represent the earliest published work on the high mannose oligo-saccharide receptor in hepatic non-parenchymal cells.

In 1976 John Baynes joined the University of South Carolina as an Assistant Professor in the Dept. of Chemistry and Biochemistry and carried out research in five areas. Four of these were intertwined and addressed the chemical biology of postsynthetic modification of proteins by the Maillard reaction in aging and age-related disease, the chemical nature of carbonyl and oxidative stress in diseases such as diabetes, obesity, atherosclerosis, cataract and chronic inflammatory diseases. Notable achievements include the discovery, in 1986, of the first genuine advanced glycation endproduct (AGE), $\mathrm{N}^{\varepsilon}$-carboxymethyl-lysine (CML), later also identified as an advanced lipoxidation endproduct (ALE). These discoveries profoundly impacted in the field, resulting to date in over 1500 citations combined for both papers [1,2].

The significance of John Baynes' work, however, reaches far beyond these semantic discoveries. Not only did he point out the necessity of developing chemically well understood molecular markers of protein damage for the study of metabolic and inflammatory diseases of aging, but he played a pioneering role in proposing that glycation, oxidation and lipid peroxidation are part of a broader "glyco/lipoxidative" stress concept in diabetes. This concept was formulated in two brilliant reviews that became citation classics [3, 4], each reaching over 3800 and 2600 citations, respectively. Equally groundbreaking were the discoveries of several novel advanced glycation structures as well as protein adducts and crosslinks previously not known to occur in vivo, including carboxyethyl-lysine (CEL), glyoxal lysine dimer (GOLD), and methylglyoxal lysine dimer (MOLD) [5, 6]. In addition to these discoveries, Baynes and colleagues not only characterized the pharmacological and chemical properties of pyridoxamine as an diabetic anti-complication agent [7], but he also contributed to debunking misconcepts about the pharmacological properties of AGE breakers, such as alagebrium. In particular, he and coauthors pointed to the need to consider that diabetic complications might be linked to redox-active catalytic metals, such as $\mathrm{Cu} 2+$, since they found that many anti-complication agents also have chelating properties $[8,9]$.

In the past 10 years, John Baynes and colleagues' work took on a new twist with the discovery that fumarate can bind reactive sulfhydryl groups to generate a succinyl adduct [10]. This innovative discovery is important because "protein succination" links protein damage to the TCA cycle and mitochondrial metabolism, as recently reviewed by Frizzell [11].

John's superb achievements were recognized in many ways throughout his career, most notably in form of a prestigious MERIT award for research on "Glycation of Protein in Diabetes" from the National Institutes of Health. This award provides long-term research support (1986-2002), a NIH Research Career Development Award, the title of Carolina Distinguished Professorship, and multiple distinguished recognitions by the American Chemical Society and the American Diabetes Association, to name just a few.

John Baynes recently confided to friends that he is stepping back from bench work research. He leaves behind him a superb record, having no less than 190 publications, $26 \mathrm{PhD}$ students, 16 MS students, 28 postdoctoral fellows, and numerous undergraduate students. In addition, he has devoted countless hours of service to various research and peer-review organizations and editorial boards, such as those of the Journal of Biological Chemistry or Diabetes. Most recently he has been working on the 5th edition of the popular textbook Medical Biochemistry (JW Baynes \& MH Dominiczak, Eds). He has also been playing major roles in supporting Maillard reaction research by hosting and chairing the 8th International Symposium on the Maillard Reaction (2004), as an officer of the International Maillard Reaction Society (IMARS), and as a fundraiser and editor of numerous proceedings of the international Maillard Reaction symposia. He has also assisted with peer-review of several of the papers presented below.

Finally, John Baynes would be the first to acknowledge the supportive role his long-time coworker and wife, Suzanne R. Thorpe, $\mathrm{PhD}$, (Distinguished Research Professor Emeritus, Univ. of South Carolina) played in many of the accomplishments enumerated above. A superb analytical chemist in her own rights, it was always a pleasure to hear her present the 
team's work at conferences, many of which dealt with the clinical significance of AGEs/ALEs. Her special interest and expertise in lipid metabolism often added a novel and fascinating insight into the meaning of the Maillard Reaction in vivo. Also, her many contributions as an Officer of IMARS and Editor of IMARS Highlights added toward much of the progress of the field until her retirement. No doubt, John and Suzie became a much beloved "Couple scientists of the Maillard Reaction" and we hope that we will have the pleasure of their friendship and scientific wit at many future Maillard Reaction and other conferences, beyond any new goals they may have set for themselves for the years to come.

It is in this spirit that the 12th International Maillard Reaction Symposium (ISMR12) was held in Tokyo from September 1-4, 2015. Co-sponsored by the dynamic Japanese Maillard Reaction Society (President Teruo Miyazawa, Secretary Ryoji Nagai) and IMARS, it covered recent developments in the chemistry and biology of the Maillard Reaction in biology and food science.

The papers presented in this Special Issue of Glycoconjugate dedicated to "Advanced Glycation in Diabetes, Aging, and Age-Related Diseases" represent a compendium of selected papers focusing primarily on the MR in vivo, i.e. as it happens in diabetes, aging and age-related diseases. Papers dealing with the "exogenous" Maillard Reaction, such as in food, aromas, food chemistry and technology and food safety will be published in Food and Function. The first five articles are meant to review issues of general importance to the field of the Maillard reaction in vivo. Taniguchi and colleagues report on how two different types of chemistries, glycosylation vs. glycation, can impact on a single disease such as Alzheimer's. While glycation of primary amines, originally named "non-enzymatic" glycosylation, initially leads to a relatively stable Amadori product that is structurally similar to a glycosidically-linked sugar, though not in its reactivity, Glomb provides a superb update of the chemical pathways of the advanced Maillard Reaction in vivo. To date, some 15 reactive carbonyls, osones, and ketoaldehydes are known, eventually leading to more than 30 aliphatic and heteroaromatic adducts and crosslinks that modify mostly lysine and arginine in amino acids, peptides and proteins, but also purine and pyrimidine bases in nucleic acids.

The particular problem of detoxifying oxoaldehydes that form intracellularly from glycolysis is addressed by Thornalley and colleagues, whereby the investigators describe novel and potent pharmacological approaches based on glyoxalase I inducers. Yet, which diabetic patient is at risk of micro- and macrovascular disease progression will depend on sensitive and, if possible, specific diagnostic methods to determine the AGE burden. The practical solution is the noninvasive determination of skin autofluorescence with an AGE reader, such as the one pioneered by Andries Smit and colleagues who provide an update on this topic.
The issue of detecting and quantitating Maillard Reaction products in vivo has been central to the field from day one. The problem is compounded by the fact that most MR products are present in low levels, usually modifying at most $1-3 \%$ of the parent amino acid residue, while generally being poorly immunogenic. Yet, today's highly sensitive mass spectrometry instruments make it possible to quantitate femtomole levels of AGEs, as discussed by Rabbani and colleagues, while monoclonal antibody technology pioneered by Nagai and others now make it possible to perform ELISAs and immunolocalize AGEs in the tissue. Both methods have advantages and limitations, but clearly the demand for high volume as well as molecular imaging methods will only increase in the future.

In the clinical setting, the issue of which marker should be included in a panel of skin AGEs as markers for the long-term progression of micro- and macrovascular disease in Type 1 diabetes is addressed by Monnier and colleagues who determined the "pecking order" of skin AGEs as risk markers based on 25 years of data. Yet, the extent to which skin data that are applicable to Type 1 diabetes can be extrapolated to Type 2 diabetes is not yet entirely clear. Masania and colleagues make a strong point for the fact that methylglyoxal could be a mediator of dicarbonyl stress and insulin resistance in clinical obesity, necessitating innovative approaches to counteract these metabolic threats, e.g. by inducing glyoxalase 1 expression. The alternative solution, which was proposed earlier by Thornalley himself, was to utilize thiamine (or benfotiamine) to activate transketolase and thus decrease methylglyoxal levels. Now, Bartáková, Kaňková and coworkers report below that thiamine metabolic abnormalities may result in dysfunctional protection against advanced glycation in gestational diabetes, while Gurecká, Šebeková and colleagues find, among obese adolescents, that a decline in SRAGE and esRAGE precedes the development of metabolic syndrome.

The topic of RAGE receptor activation in diabetes and agerelated disease is of growing importance together with the recognition that inflammatory pathways are activated in a host of diseases in which chronicity is a problem. In that regard, RAGE is a pleiotropic molecule, i.e. likely beneficial when activated by acute inflammation, but detrimental when activated by endogenous ligands such as CML, (perhaps also CEL and MG-H1), high mobility group proteins (HMGB1), some of the S100 A proteins and other protein ligands linked to aggregation. In rodent studies, three articles report on the role of RAGE in disease, namely how dietary AGEs impact on RAGE and the heart (Neviere, Boulanger et al.), how RAGE and glyoxalase expression are intertwined in kidney disease (Inagi), and how lens epithelial mesenchymal transition is triggered by AGE-RAGE (Raghavan \& Nagaraj). The extent to which glyoxalase plays a role in these processes is further suggested by the data from Bouwers and Schalkwijk who find that glyoxalase-1 overexpression partially prevents diabetes-induced impaired arteriogenesis in a rat hindlimb 
ligation model. Finally, Borg and Forbes took on to review existing data on the elusive AGER1 receptor that is identical with oligosaccharide transferase OST-48. This receptor is considered to have clearance properties for circulating AGEs, but as the authors point out, it also has important signaling properties. They conclude that more data is needed to fully comprehend this elusive aspect of circulating AGEs.

The final chapters of this Special Issue are dedicated to pharmacological interventions against the Maillard Reaction in vivo. A splendid review of the "traditional" pharmacological approaches is provided by Aowen and Forbes, while West and coauthors review anti-aging interventions using "iridoids", i.e. AGE inhibitors that are present in natural products such as the noni fruit. In contrast, Yamagishi and colleagues present intriguing data suggesting that DNAaptamers, which are a class of small nucleic acid ligands that are composed of single-stranded RNA or DNA oligonucleotides and have high specificity and affinity for their targets, could be used to clinically to trap AGEs and therefore block RAGE activation.

Finally we wish to thank all those whose work contributed to the birth of this Special Issue dedicated to glycation and the Maillard Reaction in vivo. First, special thanks go to the sponsors of ISMR12, in particular a special gift from Morinda Co as well as most generous support from various sponsors (http://www.imars.umin. $\mathrm{jp} /$ sponsors.html). We also wish to thank in particular the Glycoconjugate staff, Editor Prof. Dr. Hans Vliegenhart, Ms. Madelyn Marciano, Leonora Panday and the Production staff at Springer Co., for their help with publishing these proceedings. We finally wish to acknowledge Dr. John Baynes himself for his help with the review process of several of the 19 articles that constitute the "Special Issue".

\section{References}

1. Ahmed M.U., Thorpe S.R., Baynes J.W.: Identification of $\mathrm{N}$ epsilon-carboxymethyllysine as a degradation product of fructoselysine in glycated protein. J. Biol. Chem. 261, 4889-4894 (1986)

2. Fu M.X., Requena J.R., Jenkins A.J., Lyons T.J., Baynes J.W., Thorpe S.R.: The advanced glycation end product, Nepsilon(carboxymethyl)lysine, is a product of both lipid peroxidation and glycoxidation reactions. J. Biol. Chem. 271, 9982-9986 (1996)

3. Baynes J.W.: Role of oxidative stress in development of complications in diabetes. Diabetes. 40, 405-412 (1991)

4. Baynes J.W., Thorpe S.R.: Role of oxidative stress in diabetic complications: a new perspective on an old paradigm. Diabetes. 48, 1-9 (1999)

5. Ahmed M.U., Brinkmann Frye E., Degenhardt T.P., Thorpe S.R., Baynes J.W.: N-epsilon-(carboxyethyl)lysine, a product of the chemical modification of proteins by methylglyoxal, increases with age in human lens proteins. Biochem. J. 324, 565-570 (1997)

6. Frye E.B., Degenhardt T.P., Thorpe S.R., Baynes J.W.: Role of the Maillard reaction in aging of tissue proteins. Advanced glycation end product-dependent increase in imidazolium cross-links in human lens proteins. J. Biol. Chem. 273, 18714-18719 (1998)

7. Metz T.O., Alderson N.L., Chachich M.E., Thorpe S.R., Baynes J.W.: Pyridoxamine traps intermediates in lipid peroxidation reactions in vivo: evidence on the role of lipids in chemical modification of protein and development of diabetic complications. J. Biol. Chem. 278, 42012-42019 (2003)

8. Price D.L., Rhett P.M., Thorpe S.R., Baynes J.W.: Chelating activity of advanced glycation end-product inhibitors. J. Biol. Chem. 276, 48967-48972 (2001)

9. Frizzell N., Baynes J.W.: Chelation therapy: overlooked in the treatment and prevention of diabetes complications? Future medicinal chemistry. 5, 1075-1078 (2013)

10. Alderson N.L., Wang Y., Blatnik M., Frizzell N., Walla M.D., Lyons T.J., Alt N., Carson J.A., Nagai R., Thorpe S.R., Baynes J.W.: S-(2-Succinyl)cysteine: a novel chemical modification of tissue proteins by a Krebs cycle intermediate. Arch. Biochem. Biophys. 450, 1-8 (2006)

11. Frizzell N., Lima M., Baynes J.W.: Succination of proteins in diabetes. Free Radic. Res. 45, 101-109 (2011) 